\title{
INTRA-PULMONARY CALCIFICATION AND HISTOPLASMIN SENSITIVITY
}

BY

\author{
BRIAN H. MCCRACKEN \\ From St. Athan R.A.F. General Hospital
}

\section{INTRODUCTION}

Before the present decade it used to be assumed that the finding of calcification in the lungs was in itself almost sufficient evidence to diagnose old tuberculosis, but recent investigations in the U.S.A. have suggested that there are common causes other than healed tuberculosis for this condition. The first suggestion of a common alternative cause was made by Aronson and others (1942), who demonstrated that coccidioidomycosis was probably responsible for calcification in Indian children in the south-west of the U.S.A. Aronson investigated the relationship of pulmonary calcification to fungus infection by means of intradermal skintesting with extracts of the fungi concerned.

Palmer (1945), while studying tuberculosis in student nurses in the central eastern States, found a relatively high incidence of calcified pulmonary nodules, with negative tuberculin reactions in the majority of nurses with these lesions. Palmer found by testing these nurses with coccidioidin (an extract of Coccidioides immitis) that no relationship existed between coccidioidomycosis and pulmonary calcification in the area where he was working. Following a suggestion of Dr. C. E. Smith, of Stanford University, he next tried testing the nurses with histoplasmin, an extract of Histoplasma capsulatum, another fungus. Palmer found that many of these nurses were positive reactors, that is, the skin test produced a local reaction indicating a sensitivity to histoplasmin. He was able to show a geographical variation in the incidence of positive reactors to histoplasmin, and a similar geographical variation in the incidence of pulmonary calcification.

Christie and Peterson (1945 and 1946) published similar findings in children and students in the Mississippi Basin.

Zwerling and Palmer (1946) investigated student nurses by chest radiography and skin testing with tuberculin and histoplasmin. Of 6,199 nurses examined, 698 showed calcification; among these, 166 were tuberculin-positive and 603 were histo- plasmin-positive. Only 38 (5.4 per cent) were negative to both tests. Four times as many nurses showed calcification in Kansas as in Philadelphia, and over four times as many were sensitive to histoplasmin. Waring and Gregg (1947) examined 494 school-children in South Carolina by chest radiography and tuberculin skin-test; of the 357 tuberculin-negative children, 8 showed pulmonary calcification ; 5 of the 8 reacted to histoplasmin, and these had all resided for some time in the States where a high incidence of histoplasmin sensitivity had been demonstrated.

In these investigations the type and distribution of the calcification gave no clear indication of whether the cause was tuberculosis or not, although bilateral and widespread calcification was relatively more likely to be of the tuberculin-negative type. Although there was a high correlation between tuberculin-negative pulmonary calcifications and sensitivity to histoplasmin, it by no means follows that histoplasmosis, that is, infection with the mould Histoplasma capsulatum, is the only cause to be considered for the calcifications in the tuberculin negative subjects. Emmons and others (1945) have demonstrated that the histoplasmin skin reaction is not a specific one and that there is considerable overlap between the reactions to histoplasmin (extract from Histoplasma capsulatum), blastomycin (from Blastomyces dermatitidis), coccidioidin (from Coccidioides immitis), and haplosporangin (from Haplosporangium parvum). On the other hand the area in the central eastern States where the highest incidence of histoplasmin sensitivity has been found, and where the majority of cases of histoplasmosis have been described, is quite distinct from that area in the south-west of North America where the endemic focus of Coccidioides immitis infection exists, and where there is widespread sensitivity to coccidioidin. The former area is located in the States on each side of the central part of the Mississippi river, while the latter is largely confined to California (San Joaquin Valley Fever), Arizona, southern Utah, 
western Texas, New Mexico, the western part of Mexico, and the adjacent Central American republics (Smith, 1943). Further, Howell (1947), in experimental testing of allergens on highly sensitive animals, obtained relatively few cross-reactions between histoplasmin and blastomycin when critical titres of antigens were used.

Regardless of the specificity of the histoplasmin test, however, it is clear that in certain areas in America there is a cause other than tuberculosis for a large proportion of cases of pulmonary calcification, and it is probable that the calcification is preceded at some time by an active stage which, although benign, may simulate active tuberculosis (Zwerling and Palmer, 1947).

\section{Present Investigation}

In order to determine whether or not any significant proportion of pulmonary calcifications in this country were associated with sensitivity to histoplasmin rather than to tuberculin, a survey of Cardiff University College students was carried out in collaboration with the miniature mass radiography unit of the Welsh National Memorial Association.

\section{Methods}

A voluntary mass radiography survey of all the students in the college was carried out by the Welsh National Memorial Association, and films were taken of 1,188 students, nearly all of whom were between 17 and 28 years of age. These films were examined on a projection screen, and the films showing definite and probable calcification noted. The calcification was classified as (a) hilar, (b) peripheral, and (c) hilar and peripheral, that is, calcified primary complex.

The students whose films had shown calcification were recalled for skin testing.

The materials used for skin testing were: (1) old tuberculin 1 in 10,000 and 1 in 1,000 ; (2) histoplasmin 1 in 1,000 and 1 in 100 ; (3) oidiomycin (extracted from Monilia albicans) 1 in 1,000 and 1 in 100 ; (4) a mixed solution containing allergens from Blastomyces dermatitidis, Sporotrichum schencki, Trichophyton mentagrophytes, and Botrytis cinerea, in individual concentrations of 1 in 1,000 and 1 in 100 .

The stock histoplasmin was prepared by Dr. J. T. Duncan at the London School of Hygiene and Tropical Medicine as an autolysate by sowing two strains of Histoplasma capsulatum in flasks of suitable culture medium, incubating for six months at $22^{\circ} \mathrm{C}$., and then Seitz-filtering. Merthiolate, 1 in 10,000, was added as preservative to the dilutions for skin testing. The other allergen solutions were prepared similarly from the corresponding fungi.

The skin tests were performed by intradermal injection of about $0.1 \mathrm{ml}$. into the skin of the forearm. Most of the students were tested with all four solu- tions, but some received only tuberculin and histoplasmin. The stronger solutions were injected if no positive reactions were obtained with the weaker ones, the readings in each case being made after fortyeight hours. The accepted standard of $1 \mathrm{~cm}$. induration was applied to the reading of the tuberculin test, and would have been applied to the other tests had positive reactions been obtained.

\section{Results}

Radiographs of the chests of 1,188 students were examined, and evidence of calcification was observed in 87 ( 7.3 per cent) ; 24 of these were women (5.3 per cent of 451$)$ and 63 men $(8.5$ per cent of 737).

\section{Skin reactions}

Of the 87 cases showing calcification, 82 were subjected to skin tests: 67 were found to be tuberculin-positive and 15 tuberculin-negative ( 82 per cent positive). Table $I$ shows the numbers of students in the different groups who received injections with all the allergens, and those who received only tuberculin and histoplasmin. All the tests with histoplasmin and the other fungal allergens were negative.

\section{TABLE I}

SUMMARY OF SKIN TESTS WITH TUBERCULIN AND WITH 3 FUNGAL EXTRACTS (HISTOPLASMIN, OIDIOMYCIN, AND A MIXED EXTRACT) IN 82 STUDENTS SHOWING RADIOGRAPHIC EVIDENCE OF INTRAPULMONARY CALCIFICATIONS *

(All the tests with fungal extracts were negative)

\begin{tabular}{|c|c|c|c|}
\hline & \multicolumn{3}{|c|}{ Tuberculin skin test } \\
\hline & $\begin{array}{l}\text { Positive } \\
\text { to } 1 \text { in } \\
10,000\end{array}$ & $\begin{array}{l}\text { Positive } \\
\text { to } 1 \text { in } \\
1,000\end{array}$ & Negative \\
\hline $\begin{array}{l}\text { Tested with } 3 \text { fungal ex- } \\
\text { tracts } 1 \text { in } 1,000 \\
\text { Tested with histoplasmin } \\
\text { only } 1 \text { in } 1,000 \\
\text { Tested with } 3 \text { fungal ex- } \\
\text { tracts } 1 \text { in } 1,000 \text { and } \\
1 \text { in } 100 \\
\text { Tested with histoplasmin } \\
\text { only } 1 \text { in } 1,000 \text { and } \\
1 \text { in } 100\end{array}$ & $\begin{array}{l}33 \\
20\end{array}$ & $\begin{array}{l}7 \\
7\end{array}$ & 10 \\
\hline Total & 53 & 14 & 15 \\
\hline
\end{tabular}

In another study, a series of 62 airmen from an R.A.F. entry were voluntarily skin-tested with histoplasmin to confirm the relative rarity of positive reactors in this country, and using both 1 in 1,000 and 1 in 100 solutions, no positive reactions were obtained. 
The batch of histoplasmin used in this investigation has been subjected to guinea-pig testing by Dr. J. T. Duncan in the London School of Hygiene and Tropical Medicine, and found to have satisfactory allergenic potency. Some of the same batch of histoplasmin has been tested on human subjects, including some positive reactors to the U.S. product, by Dr. C. E. Palmer, of the U.S. Public Health Service, and has been found to give results strictly comparable with those obtained with the standard U.S..product.

\section{Types of calcification}

The 87 cases of calcification were classified into 76 with hilar calcification only, 5 with peripheral calcification only, and 6 with hilar and peripheral calcification in primary complex grouping. All the 11 cases with peripheral lesions were tuberculinpositive, and 56 out of the 76 with hilar lesions only were positive.

\section{Discussion}

No positive reaction to histoplasmin, oidiomycin, or a mixed pool of allergens from Blastomyces, Sporotrichum, Trychophyton, and Botrytis was obtained in any of the students who showed radiographic evidence of intrapulmonary calcification. The majority had a positive tuberculin reaction, and in these there is no reason to doubt the tubercular origin of the calcifications. In the 15 cases with a negative tuberculin reaction the aetiology is not established, but in view of the completely negative results with the fungal allergen tests infection with the fungi used in these tests is unlikely. The fact that all the cases with peripheral calcification were positive to tuberculin is perhaps significant, since the diagnosis of hilar calcification is more difficult, and dense shadows in the hilum are more likely to be interpreted erroneously than similar shadows more peripherally situated. Perhaps some of the 15 tuberculin-negative cases with apparent hilar calcifications were due to such errors in interpretation.

\section{Conclusion}

It seems justifiable to conclude that in South Wales, where nearly all of these students lived, skin-sensitivity to the fungus allergens used is uncommon, and that fungus infection such as histoplasmosis is not a significant factor in producing intrathoracic calcification.

\section{SUMMARY}

A brief account is given of some American investigations into the relationship between pulmonary calcification and infection with Histoplasma capsulatum and other fungi. In a survey of students in Cardiff, 1,188 miniature chest radiographs were examined, and 87 students with evidence of pulmonary calcification were discovered. In 82 of these, who were skin-tested, 67 ( 82 per cent) were tuberculin-positive, and none of them reacted to any of the extracts of fungi used, including histoplasmin. It is concluded that pulmonary calcification in South Wales is not related to sensitivity to histoplasmin or similar extracts from certain other fungi.

I wish to thank the Welsh National Memorial Association for their willing co-operation and for facilities to examine the students ; Dr. J. T. Duncan, of the London School of Hygiene and Tropical Medicine, who prepared these extracts and gave advice; the University of Wales for permission to perform the skin tests on their students ; and the Air Ministry for permission to publish this paper.

\section{REFERENCES}

Aronson, J. D., Saylor, R. M., and Parr, E. I. (1942). Arch. Path., 34, 31.

Christie, A., and Peterson, J. C. (1945). Amer. J. publ. Hlth., 35, 1131.

Christie, A., and Peterson, J. C. (1946). J. Pediat., 29, 417.

Emmons, C. W., Olson, B. J., and Eldridge, W. W. (1945). Publ. Hlth. Reps., Wash., 60, 1383.

Howell, A. (1947). Publ. Hlth. Reps., Wash., 62, 631.

Palmer, C. E. (1945). Publ. Hlth. Reps., Wash., 60, 513.

Palmer, C. E. (1946). Publ. Hlth. Reps., Wash., 61, 475.

Smith, C. E. (1943). Med. Clin. N. Amer., 27, 790.

Waring, J. I., and Gregg, D. B. (1947). Amer. J. Dis. Child., ry, 139.

Zwerling, H. B., and Palmer, C. E. (1946). Radiology, $47,59$.

Zwerling, H. B., and Palmer, C. E. (1947). J. Amer. med. Ass., 134, 691. 